\title{
Rotational Dynamics: An Exciting Challenge
}

\author{
Julio Cano \\ Advanced Dynamics C.B., Madrid, Spain \\ Email:jcano@coiim.es
}

How to cite this paper: Cano, J. (2017) Rotational Dynamics: An Exciting Challenge. World Journal of Mechanics, 7, 6884.

https://doi.org/10.4236/wjm.2017.73008

Received: January 6, 2017

Accepted: March 20, 2017

Published: March 27, 2017

Copyright $\odot 2017$ by author and Scientific Research Publishing Inc. This work is licensed under the Creative Commons Attribution International License (CC BY 4.0).

http://creativecommons.org/licenses/by/4.0/ (c) (i) Open Access

\begin{abstract}
This paper presents the reference criteria of rotational dynamics of accelerated systems in the field of Rational Mechanics, exposing the differentiation between inertial and non-inertial dynamic systems, and describes the unique research project developed by Advanced Dynamics CB up to proposing the Theory of Dynamic Interactions. This paper aims to deliver a commentary on developments in the field of rotational dynamics. Professor Barceló proposes that in today's physics, a change of mindset is necessary that allows us to accept the true behavior of mobile objects subject to non-coaxial speed variations. The proposed dynamic allows us understand certain effects of rotating bodies. The application of these dynamic hypotheses to other fields of physics and technology will possibly allow new and suggestive advances in research, especially to bodies and systems with rotation in astrophysics and astronautics, allowing new dynamic working hypotheses.
\end{abstract}

\section{Keywords}

Rotational Motion, Dynamic Interactions, Rotational Inertia, Accelerated Systems, Angular Displacement

\section{Background}

Newton's celestial mechanics conceived a new theoretical model around the knowledge of the laws of the world, generating a true scientific revolution at that time. It fuses in its model the advances achieved since the Renaissance, and determines a definitive qualitative rupture with previous thinking, based on Greek and scholastic thought. For some, it was one of the most important intellectual feats of the human species.

The influence of the Principia (Philosophiae naturalis principia matematica), extended beyond the scope of Physics itself, constituting the raison de être of the Natural Philosophy of the Enlightenment. [1]

In Newton's case, his recognition was exceptional, and it was not late. It is 
endorsed by the epitaph found on his own tomb in Westminster Abbey (1727): Here lies Sir Isaac Newton, Knight, who by a vigour of mind almost supernatural, first demonstrated, the motions and figures of the planets, the paths of the comets, and the tides of the oceans... Let Mortals rejoice that there has existed such and so great an ornament of Nature.

Later Einstein proposed a new image of nature, totally different from the previous one, and even proposes that its conception replaces the Newtonian one. Consensus is rapidly spreading in the scientific community, but in spite of this, the Newtonian model still maintains its place in society's subconscious. And not only in the social subconscious but also in that of engineering and in multiple scientific sectors, where the Newtonian model continues to be applied right up to today.

In his youth, Dr. Barceló warned of this strange duality while simultaneously studying physics and engineering.

\section{Classical Mechanics}

The mechanics of the solid rigid body is the physics, which studies the movement and equilibrium of solid materials, ignoring their possible deformations. For this purpose, Classical Mechanics has conceived a theoretical mathematical model, called rigid solids. This model is conceived as a set of points in space whose distances between each other are not altered by any transformation.

Dynamics is the branch of mechanics that deals with the motion and equilibrium of systems under the action of forces and describes the evolution over time of physical systems in relation to the actions that cause its changes of state. [2].

We understand motion as any change in place of a body in time with respect to another reference.

In this area, Rotation is any change of orientation of a rigid solid, or of a reference system, in which a point of the same, or a line, remain fixed.

It was estimated up to now that Classical Mechanics and its dynamics were disciplines based on foundations and that they determined a sector of structured and closed rational knowledge. However, Professor Barceló, in his book: New Paradigm in Physics, proposes new horizons in this area. In an earlier text, he already stated: The behavior of material systems exposed to external actions, the generation of spatial rotation and the quantities conserved associated with such circumstances have been widely debated, but we believe that this analysis can still be subject to new scientific and technological development based on the new hypothesis... [3]

In these texts, we will refer to the conceptual adventure developed by Dr. Barceló and his Advanced Dynamics research team, in the search for new solutions to interpret and predict the behavior of rotating bodies, and especially, to justify the response of rigid solids, when subjected to simultaneous or successive excitation that forces them to perform rotations on different axes. 


\section{Rotational Dynamics of Accelerated Systems}

This issue of the World Journal of Mechanics is dedicated specifically to Rotational Dynamics. The first article published by Dr. Barceló in English was also in this same journal, in 2012 (See Figure 1): Analysis of Dynamic Fields in Noninertial Systems. [4] (http://dx.doi.org/10.4236/wjm.2012.23021).

Subsequently, other articles were published, with criteria and results from similar or equivalent experimental tests performed by other authors. [5] In this special issue, we are going to refer to the research work of the Advanced $D y$ namics group, and in particular to Gabriel Barcelós new book: New Paradigm in Physics, in which he describes the results obtained in his research on rotational dynamics and his proposal for a new Theory of Dynamic Interactions.

In his book, the author himself tells us about the characteristics of his research work: On the basis of certain dynamic assumptions and a new interpretation of the behavior of rotating bodies, i.e. bodies endowed with intrinsic angular momentum, when exposed to successive torques that are non-coaxial with their intrinsic rotation, we have developed new dynamic hypotheses that enable us to conclude that it is possible to configure a new mathematical model in the dynamic theory of rotating fields.

This new model enables us to explain certain behavior which has not hitherto been sufficiently understood. By means of this new conceptual model, different results are obtained for certain circumstances, exclusively based on a new interpretation of the composition or superposing of movements caused by the external actions, for example, of the momenta of forces. [6]

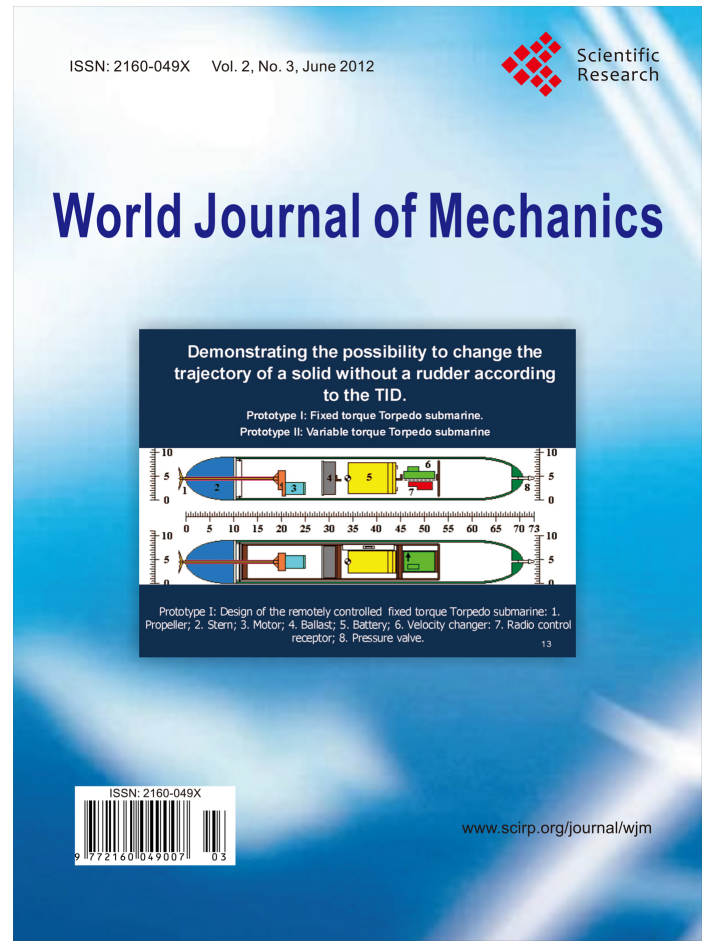

Figure 1. World Journal of Mechanics Vol.2 No.3, June 21, 2012. 
Starting from certain indications, doubts and conjectures regarding the movement of bodies endowed with intrinsic rotation, Professor Barceló has been undertaking a private research project on rotational dynamics, carrying out an analysis of the dynamic behaviour of rotating bodies: the aporia of orbiting and rotation and the observance of the dynamic equilibrium of the universe raised questions for us as regards accepted rotational mechanics, given that the aforementioned age-old dynamic equilibrium did not seem compatible with a Newtonian physics in which the forces generate constantly accelerated translation movements.

As a result of this research, hypotheses have been conceived in relation to the behaviour of bodies endowed with intrinsic angular momentum that have led to the design of an alternative mathematical model in rotational dynamics.

Both the starting hypotheses and the alternative mathematical model have been confirmed by experimental tests, as a physico-mathematical model simulation of this behaviour has been carried out.

On the basis of a reinterpretation of the behaviour of bodies endowed with intrinsic angular momentum, when subjected to successive torques, according to the hypotheses proposed, and the experimental tests performed, general laws of dynamic behaviour have been deduced. The latter were published by Dr. Barceló in this journal, (http://dx.doi.org/10.4236/wjm.2013.39036). [7]

These Laws of Rotational Dynamics are based on the inertial impossibility of matter, in certain circumstances, to modify its dynamic state, proposing the concept of rotational inertia, as an invariant of mass. These laws are conceived as a denial of the nature of selective and discriminant coupling hitherto recognized by Classical Mechanics, and allow us to conceive of an alternative Theory of Dynamic Interactions specific to bodies endowed with intrinsic rotation.

Through the generalization of the concept called "Gyroscopic torque" as a dynamic interaction torque, new and simple movement equations are obtained for bodies endowed with intrinsic angular momentum when they are provoked by new non-coaxial torques. The simplicity of these new movement equations in the field of Rotational Dynamics contrasts with the complexity of the different formulations used to date. The result is a simple mathematical formulation, which can be defined in intrinsic coordinates, without requiring an external Cartesian referential system.

\section{Rotational Dynamics of Rigid Solids}

When a real object rotates around an axis, it presents some peculiar characteristics, different from the ones that appear in translation movements, so that this movement cannot be analyzed in infinitesimal objects, or in those that may be assimilated to a particle or to a point. We cannot assign the attribute of orientation to a point, only the attribute of translation. On the other hand, in a rigid solid, at any moment, different parts of the body have different velocities and accelerations, since it can rotate, or even move in a non rectilinear uniform translation, and therefore, its orientation can evolve over time. Therefore, in ro- 
tational dynamics it is necessary to consider real objects as solids, with large numbers of particles, each with its own dynamic magnitudes, such as velocity, or acceleration.

Rotational dynamics could not have evolved, if the object of movement were a point or a particle. The Newtonian abstraction of replacing the body with its center of mass, allowed for a great easing regarding formulations in classical dynamics, but it prevented the development of rotational dynamics.

\section{Rotational Inertia}

But this peculiar behaviour of bodies in rotation establishes that, although there are no momentums or torques, if the body rotates, they are constantly generating accelerations, without external force. This apparent paradox is proposed by Dr. Barceló to introduce his concept of Rotational Inertia: $A$ different approach and a conceptual study are required for rotating bodies, given that rotational inertia is fundamentally different to translational inertia. By observing four-dimensional rotational inertia with spin tensor constant components, we can intuit an accelerated inertia movement without the existence of a constant external force.

We are going to express a paradoxical analogy that will enable us to define the concept of rotational inertia: When a body with intrinsic rotation is not subject to external forces (or their moments), in accordance with Newton-Euler equations for mechanics, if.

$$
d(I \omega) / d t=0
$$

This gives: $\omega=$ Const.

where 1 is the body's moment of inertia and $\omega$ is its angular velocity. Therefore, we deduce that the angular velocity will remain constant, even eternally, on account of its inertia. This is an experimentally proven fact.

Notwithstanding, any rotation is accelerated movement, given that the linear velocity of each particle of the rigid body, even though it remains constant, will constantly be changing position. But, $\omega$ being constant, we find ourselves faced with the contradiction of having as an example a rotation movement accelerated by inertia, without any external force. This enables us to assume the existence of a rotational inertia basically different to translational inertia.

This paradox no longer holds when we realise that we are starting from a non-inertial situation: we are referring to a body in rotation, but we are applying to it-to a moving body accelerated by rotation-the preconceived ideas of inertial systems that only apply to bodies in uniform translation. The foregoing thus enables us to reiterate the discrepancy of the dynamic behavior between bodies under inertial and non-inertial conditions, the substantial differences of which are quite often overlooked, even in physics. Accordingly, it should be noted that it is not only the reference systems that have the quality of being inertial or not, but also the bodies themselves, depending on the conditions of their movement. [8]. 
For Dr. Barceló, rotational dynamics is based on this peculiar attribute of Rotational Inertia, which assumes that bodies in rotation are necessarily systems accelerated by rotation and, therefore, they cannot be ruled by the laws of Classical Mechanics, which refer exclusively to inertial systems.

\section{Inertial and Non-Inertial Dynamic Systems}

Dr. Barceló, analyzes this dynamic differentiation in detail: Inertial and non-inertial systems were investigated in our study to better understand the response of rigid bodies subject to simultaneous, non-coaxial rotations. We understand Inertial Systems as those the reference frames of which are not subject to accelerations and in which the laws of motion put forward by Newton are observed. In them, therefore, the variation of the linear momentum is equal to the real forces that have acted on the system.

Consequently, non-inertial systems shall refer to those that are subject to variations in their velocity. This acceleration may be due to different causes.

$A$ change in the translation velocity module (linear acceleration).

$A$ change in the translation velocity direction (non rectilinear uniform translation).

An intrinsic rotation movement.

A combination of any one of the foregoing.

Therefore, non-inertial systems will be those that move with acceleration, such as, for example, those subject to changes in their velocity, like a circular movement or rotations. A moving body will be under non-inertial conditions when, being subject to null resultant external forces, nonetheless, its movement is not uniform.

The motion regularities stated by Newton will not be observed, thus in classical mechanics, in addition to the real forces, fictitious forces are introduced in these cases, to be able to explain the real observed behavior. This apparent discrepancy between reality and the theory of dynamics is, we believe, difficult to accept.

As a result of this analysis, I have put forward new dynamic hypotheses that require the extending of these studies to the theory of fields, with a view to explaining the real observed behavior in those bodies. [9]

\section{A Unique Research Project}

New Paradigm in Physics seeks to arouse our curiosity about scientific knowledge, and its author suggests that maintaining an inquiring frame of mind allows us to enhance inquisitive thought, in the confidence that observation, experimentation and reasoning enable us to question natural phenomena, or even paradoxes, axioms or principles, that may have generated doubt or confusion.

Today, in Dr. Barcelós opinion, anyone with very few resources can access new information technologies, which his parents did not know about. Computers and the Internet are instruments of work unknown to previous generations. Therefore, he concludes that a new era of human knowledge has begun. This era 
is characterized by the fact that any individual who is inquisitive can participate in this cultural development of society and can disseminate it quickly without any cost. Encouraging curiosity and participation in this new social and scientific challenge would be a desirable goal. Dr. Barceló suggests the adoption of an inquisitive attitude, which encourages the development of habits of inquiry, experimentation, research, comparison and inference, to reach a process of rational deduction and self-correction.

This challenge can be assumed by anyone who has cultural or scientific concerns, or who raises any rational question, since there are many problems that science has still to solve, or whose current interpretation generates uncertainty.

In this environment, and from the aporia between rotation and orbiting, as expressed in his book New Paradigm in Physics, Dr. Barceló has for more than forty years been developing the mentioned research program on Rotational $D y$ namics, as he observed how, in nature, the correlation by which bodies that rotate on their axis simultaneously orbit is constantly produced: $A$ mathematical expression has not been put forward to date in mechanical laws to define that possible physical correlation between orbiting and intrinsic rotational movements. However, the aporia, that there might be such a physico-mathematical relationship between both movements, can be intuitively proposed.

Moreover, we can ask ourselves if the currently known laws of dynamics to define the behavior of bodies in space are sufficiently accurate or if, on the contrary, they do not describe all the possible circumstances of the physical reality of our world with complete precision.

Neither are we aware of any type of analysis or study on simultaneous phenomena which could determine possible inferences of one phenomenon on the other. Nonetheless, the observable fact is that in the universe all orbiting celestial bodies have intrinsic rotation.

For that very reason, I set about investigating if there might be a physical nomological [10] correlation between both movements, and if so, to determine the mathematical expression of the same.

The dynamic laws hitherto accepted to determine the behavior of rotating bodies would not suffice to accurately describe the true physical reality of the rotational motion of bodies. [11]

This phenomenon, with no apparent answer to date, can be observed both in the cosmos and at the atomic level. There was no physical law to establish the justification of this behaviour of nature, whereby the orbital path of a planet or a satellite coincides temporarily with its intrinsic rotation, a phenomenon that we can interpret as also being repeated with the electron and the nucleus of atoms, as Professor Miguel Catalán liked to suggest, according to Dr. Barceló. Could we suppose that all this is the result of a coincidence? Or did it really represent natural behaviour that was not yet known, whose law was still to be stated?

The author thus reminds his professor in the book: In memory of our teacher Miguel A. Catalán Sañudo. Figure 2; Figure 3 and Figure 4 also mention Doctor Catalán. 


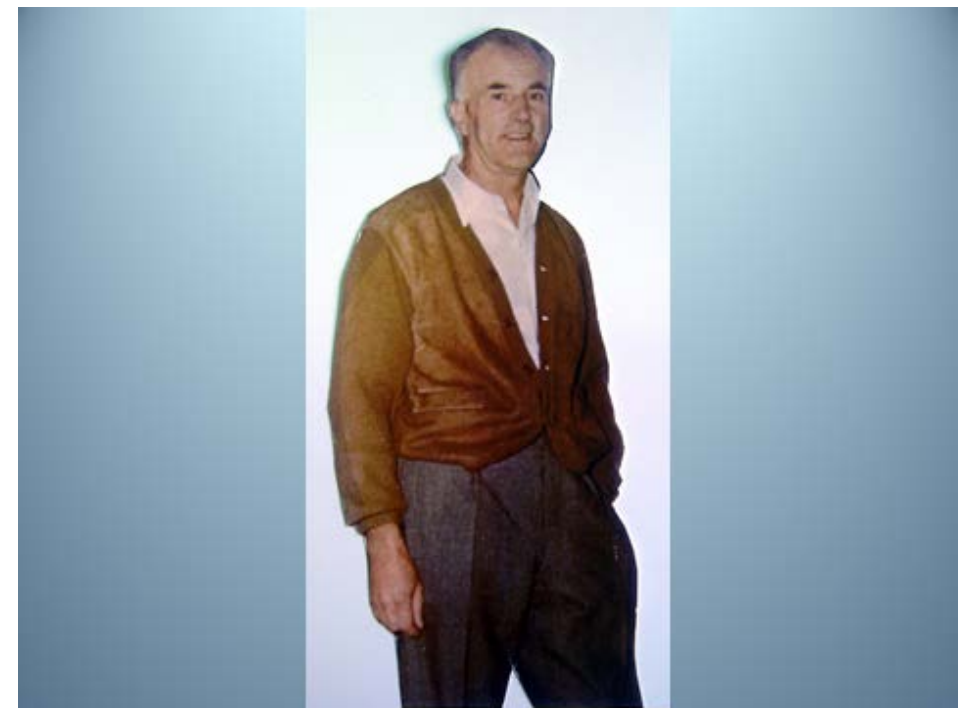

Figure 2. Miguel A. Catalán Sañudo. Spectroscopist (Zaragoza 1894-Madrid 1957) (photograph by Nicolas Urgoiti).

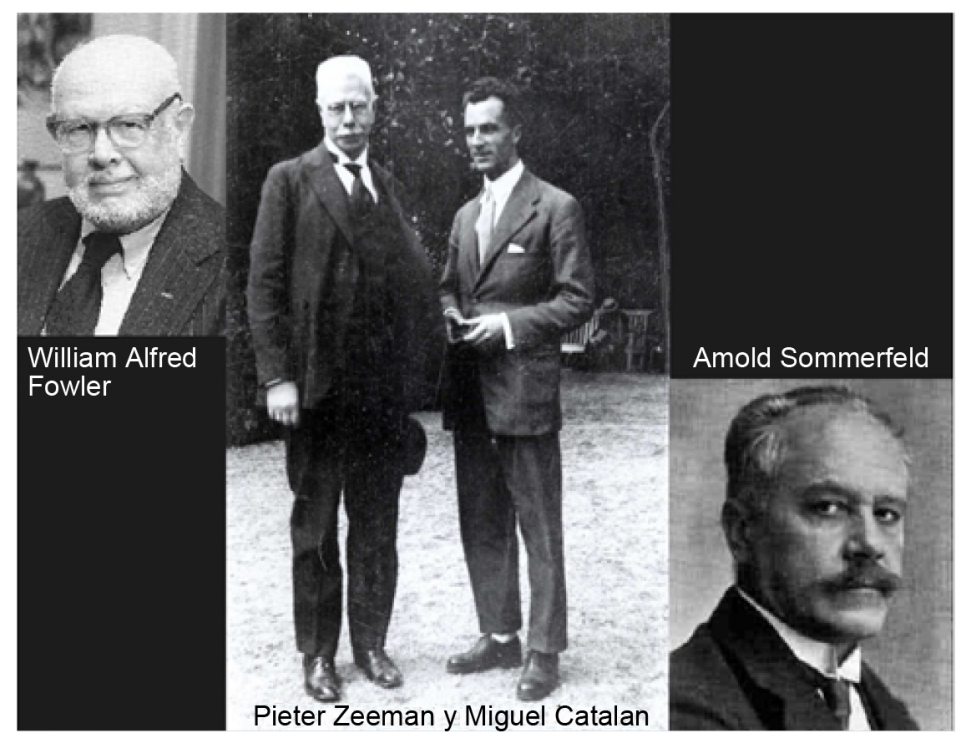

Figure 3. Miguel Catalán and Pieter Zeeman. On the left is a Portrait of William A. Fowler and on the right one of Arnold Sommerfeld, scientists connected with Catalán.

\section{Creative Curiosity}

Starting from this doubt, and from the observance of the dynamic equilibrium of the universe, Dr. Barceló began this research project with scarce resources, to explore the possible causes of these repetitive coincidences of nature and the amazing secular equilibrium of the universe: Along with the aporia of orbiting and rotation, observing the universe also caused us other new doubts: its secular dynamic equilibrium did not seem to fit in with Newtonian physics in that the forces generated constantly accelerated movements. The equilibrium of the universe and its dynamics did not seem at home in the conceptual structure of classical mechanics. [12] 


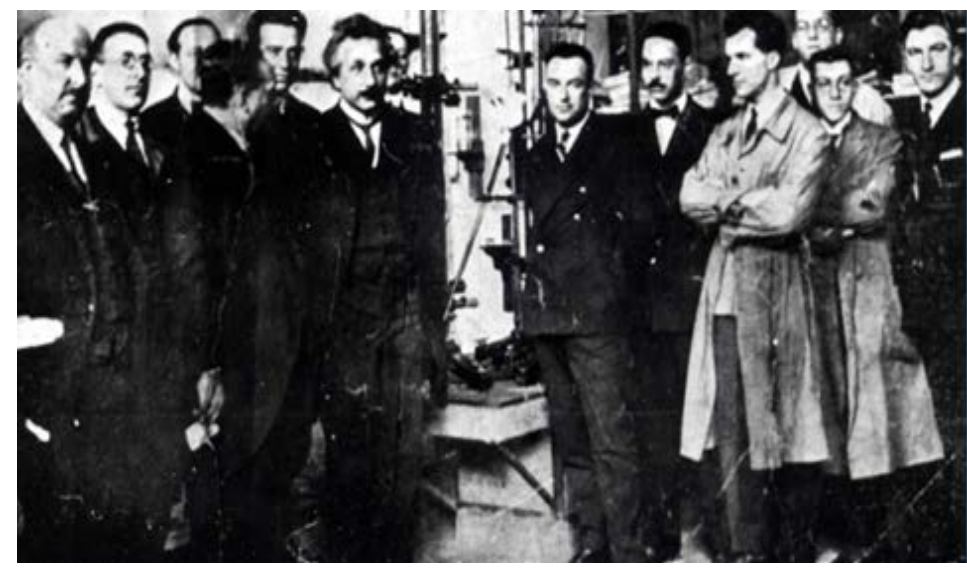

Figure 4. Einstein's visit to JAE's (Board for Advanced Studies) Physical Research Laboratory, in Madrid, where Miguel Catalán worked.

Our author wished to recover a possibly forgotten debate, but the content of which can become thrilling when studied and analyzed. In his view, there was sufficient evidence to suppose that certain studies of dynamics and rotation, begun in the 19th century, were not fully developed, being interrupted and abandoned, perhaps because of Einstein's surprising and novel theories and the far-reaching development of physics and technology that came about at the beginning of the 20th century. At that time, rotational dynamics could be forgotten in response to the fascinating challenges of physics, newly imbued with relativist and quantum theory.

We have to be aware that we live under constant temporal change, for on Earth we enjoy successive days and nights, mornings and evenings, precisely because of its intrinsic rotation, as it orbits (See Figure 5). We also enjoy the successive seasons, resulting from constantly tracing its orbiting path around the Sun.

We suggest, then, getting back in touch with these natural phenomena by recalling dynamics in non-Galilean references. We begin the analysis of the historical evolution of the dynamics of bodies in rotation, and in particular, when subjected to successive non-coaxial rotations, because if we study and experience these cases, we can see that we find ourselves with accelerated systems. It is necessary to find the new and true laws of dynamic behavior of these accelerated systems, which are not Newtonian. [13]

Even though there has been a constant evolution in human thought, Dr. Barceló has insisted in his texts that it took a long time to understand and apply the concept of rotation in scientific cosmology, so that the notion of rotational and orbiting movements of the Earth was proposed by Copernicus in the sixteenth century, and it was not until the second quarter of the twentieth century when the rotation of galaxies was accepted by the scientific community.

\section{Conjectures on Angular Displacement}

Dr. Barceló denounces the misuse of vector algebra in the modeling of rotations 


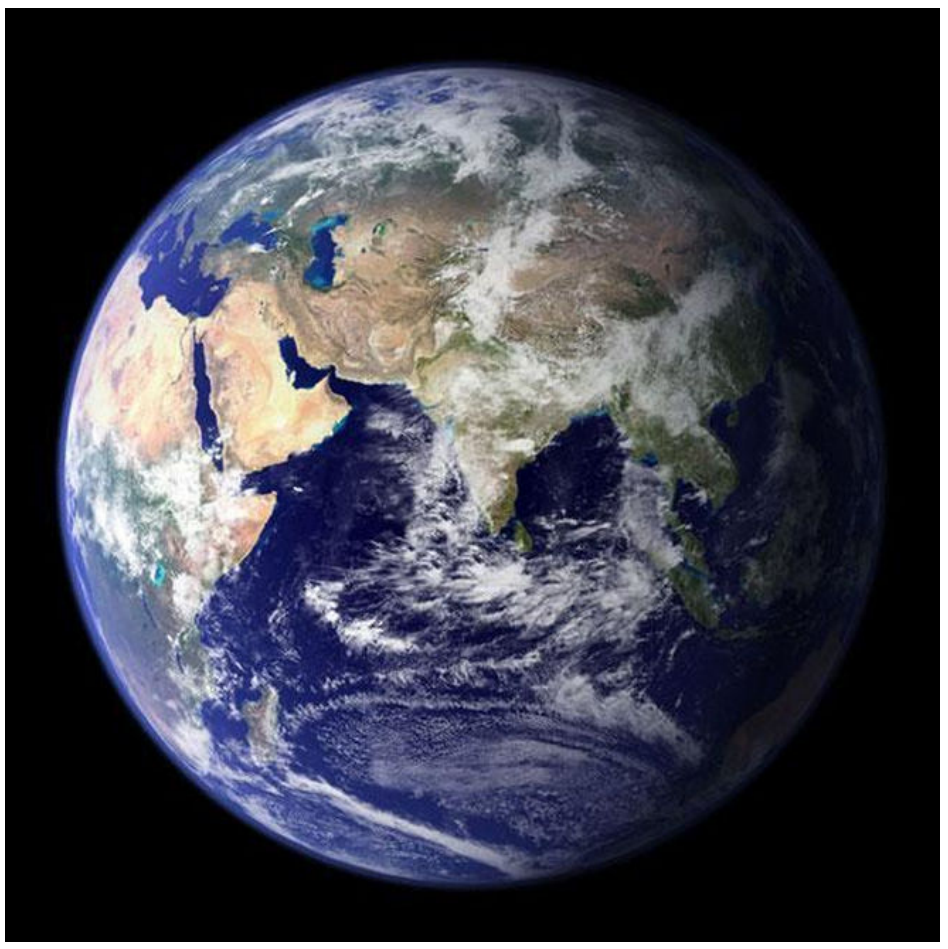

Figure 5. The Earth rotates on its axis, while simultaneously orbiting, generating our days and nights, and also our seasons.

in dynamics. He considers that errors have been generated in Classical Mechanics by not rigorously demanding the modeling of rotations and allowing the incorporation of non-inertial phenomena in their development. So the new text says: If we restrict ourselves to analyzing the phenomenon of rotation or angular displacement, understanding as such the movement of a solid around any axis that goes through it, we can propose that this magnitude fails to observe the algebraic laws of addition and commutation, as expounded below. [14]

The author recalls how certain properties of vector algebra are not fulfilled in the geometrical transformations of rotation. He puts forward a series of visual examples that demonstrate this:

\section{Angular Displacement Does Not Obey the Law of Vector Addition}

The algebraic law of vector addition states that the resultant vector of others will be a new vector determined by the so-called parallelogram or vector addition law. Consequently, if two vectors represent two different rotations, the sum of both will be a new vector that must be represented by the parallelogram law. It can be proved that angular displacement does not obey this law of vector addition.

If we take the book in Figure 6 and turn it $\pi\left(180^{\circ}\right)$, first on its vertical axis $Z$, and then $180^{\circ}$ again $\pi$ on a horizontal axis $X$, the book will end up in a different position to the one resulting from the application of a rotation equivalent to the vector sum of both rotations represented by their vectors. [15] 

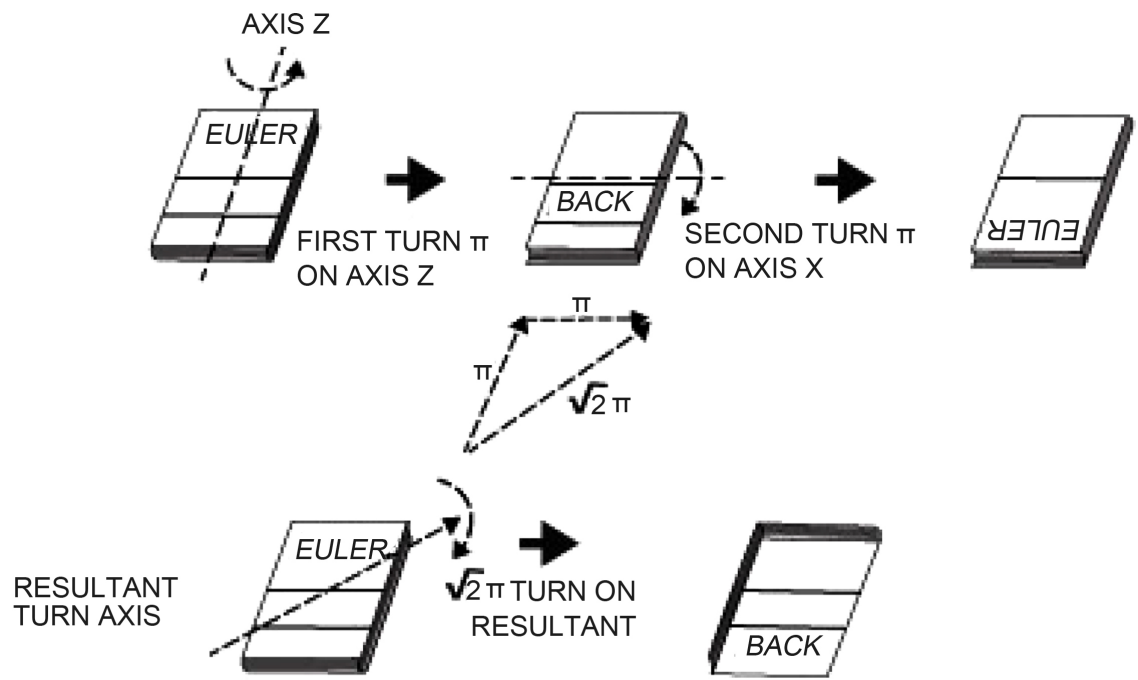

Figure 6. Angular displacement does not obey the law of vector addition [16].

\section{The Resultant Displacement Depends on the Order in Which the Movements Occur}

The rotary operations do not obey the commutative algebraic property.

$$
A+B \neq B+A .
$$

In the previous example, the book is subject to a $90^{\circ}$ rotation on its $Y$ axis (Figure 7), perpendicular to the page plane, clockwise and then is subject to another on the $Z$ axis, perpendicular the aforementioned position. With another $90^{\circ}$ rotation, the result obtained is quite different to the one obtained if we turn the book on its $Z$ axis first, as indicated, and then turn it with respect to its $Y$ axis. We see that the end result is not the same when the operations are done in reverse order. On proposing a similar example, Goldstein stated: "This conclusion, that the sum of finite rotations depends upon the order of the rotations, is strikingly demonstrated'. [17]

The result of the two angular displacements referred to external axes differs depending on the order of application. The end position of a body subject to two different angular displacements differs if these are impressed on two different axes at different successive times.

The algebraic law of commutation states that the order of the factors cannot alter the result, thus, on adding two components of the same magnitude, the result is independent of the order or succession in which they are added. Angular displacement does not obey the commutative property, given that the result of the two successive angular displacements depends on the order or sequence of the application.

It is evident that, given that observable characteristics of angular displacements, the composition of rotations is not a commutative operation. Therefore, if neither the law of addition nor vector algebra commutation is obeyed, we cannot accept the convention that an angular displacement can be represented by a vector nor, in our opinion, can vector algebra be used in rotational dynamics. 


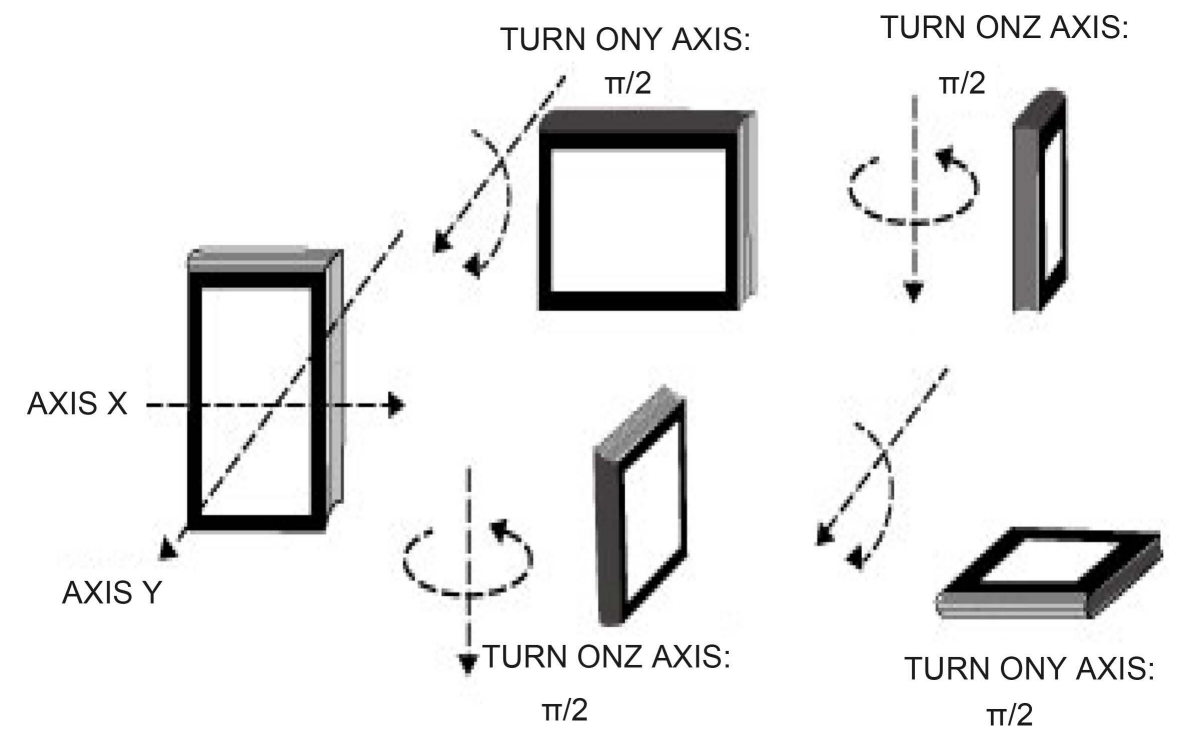

Figure 7. The result of two angular displacements referred to external axes differs depending on the order of application. The end position of the book is different after applying a quarter rotation on two different axes at different successive times [18].

The aforementioned statement seems logical, concise and beyond question and can be understood as an axiom in our analysis to determine an algebra and a system to represent rotational dynamics properly. Notwithstanding, as we will see, this statement is not universally accepted. Indeed, vector algebra has been used for many years to mathematically underpin rotational dynamics.

The question being raised is more complex, given that on analyzing this operation in depth, we notice that the laws of addition and commutation are obeyed in a particular case: when the angular displacements are coaxial, that is to say, when referring to those that occur on the same axis, which remains as the constant axis of rotation. [19]

Nonetheless, this peculiar occurrence with respect to coaxial angular displacements cannot be generalized and we must respect the true nature of rotational magnitudes. [20]

For all this, Dr. Barceló resolves his analysis by suggesting in his book that: We can propose as a conclusion that the angular displacement does not obey this law of vector addition.

The argument used in books on Classical Mechanics cannot be logically and rationally accepted as it proposes that:

1) Coaxial rotations are commutative.

2) Rotations in space are not commutative in general.

3) A finite sequence of infinitely small rotations is commutable. [21]

Therefore, he concludes that: The resultant angular displacement depends on the order in which the movements occur. [22]

Adding in his analysis of nonconformities: Dissatisfied as we were with the criteria of conventional rotational dynamics, we tried to come up with new approaches to determine the equation of motion of accelerated systems and to bet- 
ter understand and explain their behavior. After a first analysis, we became convinced that.

- Whenever a solid rigid body with intrinsic rotation is subject to an external, non-coaxial momentum, dynamic interactions are generated that cause a variation in the dynamic behavior of the body, which is not provided for in classical mechanics.

- Solid bodies with intrinsic angular momentum and a quantity of movement, when they are subject to a new, non-coaxial momentum with its rotation, change their path in accordance with a defined regularity. [23]

Dr. Barceló also studies the behavior of moving objects which are subject to several simultaneous rotations and, confirming that two rotations do not necessarily engage in a third, but rather generate two different but simultaneous rotational movements in the body, he comes to the conclusion that,...in the case of a rigid body subject to non-coaxial simultaneous rotations, (...) we can only identify the movement of each point of the body with 10 coordinates. $x_{1}, x_{2}, x_{3}$, $\varphi_{1}, \varphi_{2}, \varphi_{3}, \theta_{1}, \theta_{2}, \theta_{3}$, and time $t$, thus it has 10 degrees of freedom. [24]

\section{A Rotating World}

The first text published by the author on rotational dynamics was The Flight of the Boomerang (2006). He later published A Rotating World. [25]

In this book the author describes the deductive process followed that led to the Theory of Dynamic Interactions (TDI). It studies inertial forces and incorporates inertial reactions, also the concept of the gyroscopic torque, to the structure of a new, rotational dynamics of accelerated systems. The proposed theory generalises these dynamic concepts which had become de-structured in classical mechanics, and enables us to confirm and understand the physical and mathematical correlation that holds between orbiting and intrinsic rotation and, therefore, the reason why we have day and night on Earth (see Figure 5).

The book explains TDI rotational dynamics' hypotheses with numerous experiments and puts forward a host of behavioural situations as patent examples of it, arguing that the classical laws of mechanics, though fully valid and proven, exclusively refer to situations of translational movement in inertial systems, when in the universe and in nature, movement normally manifests itself by way of accelerations, especially in rotational dynamics.

A Rotating World is structured in four parts, with a prologue, preface, synthesis and epilogue, including as attachments: a bibliography, opinions and unpublished reports, a thesaurus and a final page of acknowledgements.

Part I: Approach

The background and objectives of this text are proposed. It apparently supports a transgressive proposal of the principles of classical mechanics, but it actually explores the dynamic behavior of non-inertial systems.

Part II: Experimental Tests

The experimental tests are described, which were performed in the last twenty years to confirm the dynamic hypotheses and their interpretation. 


\section{$\underline{\text { Part III: Suitability of Classical Rotational Mechanics }}$}

In the third part of the book an analysis is performed of the criteria underlying classical mechanics for the determination of rotational movement in solid rigid bodies in space, proposing ways to make this coherent with the experimental tests.

Part IV: Dynamics of Non-Inertial Systems

It proposes, as a result of the hypotheses and experimental methodology, a new dynamic conception for the determination of the movement of solid rigid bodies in space, endowed with intrinsic angular momentum.

Thus, the book constitutes an astonishing and fascinating contribution to the scientific world providing it with a new structure of knowledge for phenomena with rotation or non-rectilinear movement and in which accelerations are generated.

\section{Imago Universi}

This study of the evolution of human knowledge of the cosmos resulted into the book: Imago Universi, a story of human conception of the cosmos. [26]

The text describes the human evolution of the image of the universe (Imago Universi), and how human observation was generated by different and new hypothesis of the celestial dome, and, through time, the development of new mathematical models and new structures based on the logical reasoning, to understand the behaviour of the universe. It also analyses the evolution of the knowledge of rotational mechanics and its application to celestial mechanics.

The text reminds that the phenomenon of orbitation of the planets around the Sun, simultaneously with its intrinsic rotation around its axis, is not explained by Newtonian mechanics. Newton neither explains the reason for the rings of Saturn nor many systems of flat rings in our solar system like the asteroid belt, the Kuiper belt or the diffused disc. Therefore, when elaborating this dynamics theory, I intuitively acknowledged its possible application to comprehend the natural environment better, and to justify the harmony and structural stability of the universe. [27]

In its last chapter of Imago Universi, the application of the Theory of Dynamic Interactions to astrophysics and specifically to the dynamic of stellar systems and galaxies is included. The book has a video of presentation that can be viewed. [28]

It is noteworthy to underline again that in the universe, the usual and repeated movements are mainly accelerated by rotation, except those of free fall. However, the general theories of Relativity of Einstein, the relativity of Galileo-Newton and, in general, also classical mechanics, are based on the assumption of translational relativity.

These theories consider transformations as translational coordinates and therefore, when describing rotation movement, the transformation of angular (or rotational) coordinates has to be added to the transformations of translational coordinates. Moreover, the apparent inertial forces that might appear as- 
suming accelerations by rotations are not structured in those theories.

\section{Change of Mindset}

Professor Barceló proposes that in today's physics, a change of mindset is necessary that allows us to accept the true behavior of mobile objects subject to non-coaxial speed variations.

A change equivalent to that which occurred regarding the human consensus when, firstly astronauts, and then all societies viewed the Earth from space and became aware of the fragility of their environment and ecology. Then the arms race, the result of the cold war, was paralyzed by the fear that the human race could disappear, victim of its own technological and weapons successes.

In the same way, a change in the architecture of our language should occur, regardless of the language used, admitting a nature that behaves according to the dynamic criteria of TDI.

\section{Conclusions}

The experiments performed by the team of Advanced Dynamics CB demonstrate the theory proposed, but possibly the Pendulum experiment conceived by Barceló is the most remarkable, since the movement obtained in that pendulum cannot be justified under any circumstances by Classical Mechanics, while it can be by TDI.

Also, the mobile object, without traction, that slid down a slope in front of the Cathedral of Valladolid, which was carried out by Al. Pérez is an irrefutable proof of the theory.

In any case, quantitative experiments should be done, with exact measurements for a mathematical confirmation of the theory.

The present text is but a brief summary of the work done over the years in order to propose a theory of Rotational Dynamic Interactions applicable to bodies subjected to multiple external and successive torques. The initial hypotheses have been confirmed with studies and experimental tests, and with a mathematical model that allows us to simulate the real behavior of bodies that are subject to this excitation. All of which has allowed us to propose the existence of general laws of behavior in rotational dynamics, different from those of translational dynamics. A clear correlation has been obtained between the initial conjectures, the starting hypotheses, the mathematical simulation model, the deduced laws of behavior, the experimental tests performed, and the mathematical model corresponding to the equations of motion resulting from the proposed dynamic laws.

The proposed dynamic allows us to easily understand certain effects of rotating bodies, such as dynamic interactions. The application of these dynamic hypotheses to other fields of physics and technology will possibly allow new and suggestive advances in research.

Special mention should be made of the possible application of this alternative physico-mathematical model to bodies and systems with rotation in astrophysics 
and astronautics, allowing new dynamic working hypotheses.

Those interested in learning about this private research project can request more information from Advanced Dynamics C.B. or see:

http://advanceddynamics.net/; http://dinamicafundacion.com/

\section{References}

[1] Otero Carvajal, L.E. Eppur si muove, truth and knowledge. From Galileo to Stephen Hawking. University of Puerto Rico, Puerto Rico.

http://umbral.uprrp.edu/

[2] Barceló, G. New Paradigm in Physics, Volume I: Conjetures. Epilogue Volume I.

[3] Barceló, G. (2008) A Rotating World. Section 14.3, Marcombo, Barcelona, 409. http://www.dinamicafundacion.com/

[4] Barceló, G. (2012) Analysis of Dynamic Fields in Non-Inertial Systems. World Journal of Mechanics, 2, 175-180. https://doi.org/10.4236/wjm.2012.23021

[5] Pérez, L.A. (2013) New Evidence on Rotational Dynamics. World Journal of Mechanics, 3, 174-177. http://www.scirp.org/journal/wjm

https://doi.org/10.4236/wjm.2013.33016

[6] Barceló, G. New Paradigm in Physics, Volume I.

[7] Barceló, G. (2013) Theory of Dynamic Interactions: Laws of Motion. World Journal of Mechanics, 3, 328-338. https://doi.org/10.4236/wjm.2013.39036

[8] Barceló, G. (2017) New Paradigm in Physics. Section: 2.2.2-Rotational Inertia.

[9] Barceló, G. New Paradigm in Physics, Volume I: Section: 0.1-Aporia.

[10] Nomological: Relating to Nomology, Nomology: Science of Laws and Their Interpretation. Treatise on Establishing Laws, Precepts or Principles.

[11] Barceló, G. (2017) New Paradigm in Physics. Section: 0.1-Aporia. 39.

[12] Barceló, G. (2017) New Paradigm in Physics. Section: 0.1-Aporia. 42.

[13] Barceló, G. (2016) A Unique Research Project: Rotational Dynamics of Accelerated Systems. Journal of Madrid. http://jsanchezmingo.blogspot.com.es/2016_11_01_archive.html

[14] Barceló, G. (2017) New Paradigm in Physics. Section: 2.2.9-Applicable Algorithms.

[15] Barceló, G. (2006) The Flight of the Boomerang. Section 11.5-Marcombo, Barcelona, 257. http://www.dinamicafundacion.com/

[16] Barceló, G. (2017) New Paradigm in Physics. Section: 2.2.9.0-Conjectures on Angular Displacement, Figure 2.8.

[17] Goldstein, H. (1994) Classical Mechanics. Section 4.8, Reverté, Barcelona.

[18] Barceló, G. (2017) New Paradigm in Physics. Section: 2.2.9.0-Conjectures on Angular Displacement, Figure 2.9.

[19] Barceló, G. (2006) The Flight of the Boomerang. Section 11.5, Marcombo, Barcelona, 256. http://www.dinamicafundacion.com/

[20] Barceló, G. (2017) New Paradigm in Physics. Section: 2.2.9.0-Conjectures on Angular Displacement.

[21] Scala Estalella, J.J. (1995) Treatise on Vectors, Volume I, Vector Analysis, Heading 19, Editorial Síntesis.

[22] Barceló, G. (2017) New Paradigm in Physics. Section: 2.2.9.2-Vector Commutation Law.

[23] Barceló, G. (2017) New Paradigm in Physics. New Deductions, Epilogue, Volume I. 
[24] Barceló, G. (2008) A Rotating World. Chap. 7, Marcombo, Barcelona, 201.

[25] Barceló, G. (2008) A Rotating World the Theory of Dynamic Interactions. Marcombo, Barcelona, $436 \mathrm{p}$.

[26] Barceló, G. (2013) Imago Universi, a Story of Human Conception of the Cosmos. (A History of the Human Conception of the Cosmos). Arpegio. Barcelona. http://www.editorialarpegio.com/ http://imagouniversi.com/

[27] Barceló, G. (2013) Proposal of New Criteria for Celestial Mechanics. International Journal of Astronomy and Astrophysics, 3, 385-391. https://doi.org/10.4236/ijaa.2013.34044

[28] Sánchez-Blanco, J. Imago Universi Video. http://vimeo.com/62247544

\section{Submit or recommend next manuscript to SCIRP and we will provide best} service for you:

Accepting pre-submission inquiries through Email, Facebook, LinkedIn, Twitter, etc. A wide selection of journals (inclusive of 9 subjects, more than 200 journals)

Providing 24-hour high-quality service

User-friendly online submission system

Fair and swift peer-review system

Efficient typesetting and proofreading procedure

Display of the result of downloads and visits, as well as the number of cited articles Maximum dissemination of your research work

Submit your manuscript at: http://papersubmission.scirp.org/

Or contact wjm@scirp.org 